\title{
RADIOGRAFÍA DE LOS GABINETES MINISTERIALES EN BRASIL Y PERÚ (2016-2018). UN ANÁLISIS COMPARATIVO*
}

\author{
Radiography of ministerial cabinets in Brazil and Peru (2016-2018). \\ A comparative analysis
}

Inés Nercesian** y Roberto Cassaglia***

\section{RESUMEN}

A partir del triunfo de fuerzas políticas con perfil y orientación derechista en varios países de América Latina, las ciencias sociales retomaron un debate histórico acerca de los grupos de poder y el modo en que estos ejercen la dominación política. Este artículo analiza comparativamente los gobiernos de Michel Temer (2016-2018) en Brasil y Pedro Pablo Kuczynski (2016-2018) en Perú. Aunque en ambos países se asumieron programas de gobierno de orientación favorable hacia el mercado, las características y el perfil de reclutamiento de las y los funcionarios en cada país presentan una diferencia notable: en Brasil el gabinete mostró rasgos preponderantemente políticos, mientras que en Perú el cuerpo ministerial tuvo un sesgo hacia la mayor presencia de tecnócratas y figuras provenientes de la órbita empresarial. Esta investigación asume una perspectiva que se ubica en las fronteras de la sociología histórica y la sociología política y analiza el perfil del gabinete ministerial en ambos países a partir de tres variables: perfil educativo, sociopolítico y ocupacional. El artículo analiza una base de datos de elaboración propia acerca de la trayectoria educativa y ocupacional de altos funcionarios y funcionarias, así como también, estudia un conjunto de entrevistas en profundidad a informantes clave, realizadas para esta investigación. A partir del análisis comparado sostenemos que las variables de larga duración, tales como la fortaleza o debilidad de las instituciones estatales y los partidos políticos son una clave para

\footnotetext{
*Este artículo presenta resultados parciales del proyecto de investigación UBACYT20020170200271BA "Estado, élites y grupos económicos en América Latina (2008-2017)”, dirigido por Inés Nercesian, en el Instituto de Estudios de América Latina y el Caribe, Facultad de Ciencias Sociales.

${ }^{* *}$ Dra. en Ciencias Sociales, Magíster en Investigación en Ciencias Sociales y Lic. en Sociología. Investigadora Adjunta del Consejo Nacional de Investigaciones Científicas y Tecnológicas (CONICET). Profesora Adjunta en la materia Sociología Política de América Latina, Facultad de Ciencias Sociales de la Universidad de Buenos Aires (UBA). Directora del Observatorio Electoral de América Latina. Buenos Aires, Argentina. Correo electrónico: inercesian@ gmail.com

*** Sociólogo por la Facultad de Ciencias Sociales, Universidad de Buenos Aires. Maestrando en Estudios Sociales Latinoamericanos. Becario doctoral del Consejo Nacional de Investigaciones Científicas y Tecnológicas (CONICET), integra el Observatorio Electoral de América Latina. Buenos Aires, Argentina. Correo electrónico: robertocassaglia@gmail.com
} 
comprender las diferencias en el reclutamiento de los elencos ministeriales de ambos países.

Palabras clave: élites, política, Estado, Brasil, Perú

RECIBIDO: Diciembre 2018

ACEPTADO: Abril 2019

\section{ABSTRACT}

Since the triumph of political forces with a wright wing profile and orientation in several countries in Latin America, within social sciences the debate about power groups and their way of political domination was reinstated. This article analyses comparatively two government experiences: Michel Temer (2016-2018) in Brazil and Pedro Pablo Kuczynski (2016-2018) in Peru. Even though in both countries government policies with a favorable orientation towards the market were assumed, the profile and characteristics of the official's recruitment present a notable difference: in Brazil the cabinet showed more political traits, while in Peru the ministerial body had a corporate and technocratic bias. From a perspective located in the boundaries between political and historical sociology, the survey analyses the ministerial cabinet's profile in both countries starting from three variables: scholar, socio-political and occupational profiles. This work is based on own elaborated data about the educational and occupational trajectory of the high officials as well as a number of interviews with key informants. From the comparative analysis we sustain that long-lasting variables, such as the strength or weakness of the state institutions and the political parties are the key to understand the differences between the ministerial cabinet's recruitment in both countries.

Key words: elites, politics, State, Brazil, Peru.

\section{Introducción}

A partir del triunfo de fuerzas políticas con perfil y orientación derechista en varios países de la región, se repuso un debate histórico en las ciencias sociales sobre los grupos de poder en América Latina. Aun cuando pudieran hallarse elementos comunes, existen continuidades y rupturas en los distintos países: en algunos casos, gobiernan funcionarios y funcionarias con un perfil empresarial, en otros, una clase política y un funcionariado con un perfil de mayor desempeño en la órbita pública. Este artículo se inscribe en un proyecto de investigación más amplio cuyo objetivo es analizar los modos de ejercicio del poder político de las élites en América Latina, a 
Radiografía de los gabinetes ministeriales en Brasil y Perú (2016-2018). Un análisis comparativo

partir de la siguiente evidencia: desde el año 2000, por lo menos ocho países son gobernados por figuras estrechamente vinculadas al mundo empresarial. ${ }^{1} \mathrm{En}$ este artículo presentamos dos de esas experiencias políticas en una clave de análisis comparativa: Michel Temer (2016-2018), en Brasil y Pedro Pablo Kuczynski (20162018). Michel Temer es un hombre que proviene del mundo de la política, como parte del histórico Partido Movimento Democrático Brasileiro (PMDB) y su gobierno marcó una ruptura respecto de las políticas implementadas por el Partido dos Trabalhadores (2003-2016). El 29 de octubre de 2015, el PMDB publicó el documento Uma pomte para o futuro, donde retomó las demandas de las corporaciones empresarias y planteó cuál era el rumbo que debía seguir la economía. ${ }^{2}$ En Perú, Kuczynski, por su biografía laboral y política, delineó un perfil tecnocrático y liberal, con fuertes vínculos con el mundo del empresariado. En ambos casos hubo una exaltación del perfil de los "expertos", quienes ocuparían los gabinetes ministeriales. Temer aseguró que había formado un "gobierno de notables" y, tiempo después, en marzo de 2018, sostuvo en el canal oficial del Planalto que se trataba del "mejor gobierno de los últimos años". Por su parte, el gobierno peruano sostuvo que había conformado "un gabinete de lujo, de la mejor gente de todo el Perú".

Este artículo es parte de una investigación comparativa acerca de los perfiles de funcionarios y funcionarias de gobiernos con sesgo tecnocrático y empresarial en América Latina, desde el año 2000 hasta la actualidad. La investigación utiliza una base

\footnotetext{
1 Nos referimos a los casos de: Vicente Fox en México, Álvaro Uribe Vélez (2002-2010) en Colombia, Elías Antonio Saca González (2004-2009) en El Salvador, Sebastián Piñera (2010-2014/2018-) en Chile, Horacio Cartes (2013-2018) en Paraguay, Mauricio Macri (2015-) en Argentina, Pedro Pablo Kuczynski (2016-2018) en Perú y un caso emblemático, Ricardo Martinelli (2009-2014) y Juan Carlos Varela (2014-) en Panamá. En Brasil, aunque Michel Temer (2016-2018) es un hombre que proviene de la histórica clase política, sus estrechos vínculos con el empresariado lo convierten en un caso que merece ser estudiado.

2 Entre las políticas se destacan dos cuestiones sensibles para el mundo empresarial: la regulación del gasto fiscal (PEC No. 241/16) ${ }^{2}$ y la reforma sobre las leyes de trabajo, conocidas en Brasil como Consolidação das Leis do Trabalho (CLT). La CLT es la legislación laboral promulgada por Getúlio Vargas el 1ำ de mayo de 1943, durante el período denominado Estado novo (1938-1945). El 13 de julio de 2017 fue modificada mediante la ley No. 13.467 y se hizo efectiva a partir de noviembre del mismo año. Veáse: Uma ponte para o futuro (29 de octubre de 2015). Fundaçao Ulysses Guimarão. Brasilia, Brasil.
} 
de datos de elaboración propia acerca del perfil de ministros y ministras de los distintos gabinetes. ${ }^{3}$ Aquí analizaremos las experiencias de Brasil y Perú, las cuales presentan diferencias notables: el gabinete brasileño muestra una mayor presencia de funcionarios y funcionarias con trayectoria educativa y ocupacional desarrollada en la órbita pública, mientras Perú evidencia mayor cantidad de figuras en el gabinete con una trayectoria ocupacional y educativa desarrollada en la órbita privada. Asimismo, se realizaron entrevistas en profundidad a informantes clave a miembros de los gabinetes ministeriales y a periodistas e investigadores especializados. Por tratarse de funcionarios que hacía muy poco habían concluido sus funciones y se requería conservar su anonimato, se preservó la identidad. La investigación asume una perspectiva ubicada en las fronteras de la sociología histórica y la sociología política. Desde este enfoque, la comparación busca analizar hechos únicos coyunturales (en este caso la constitución de dos perfiles ministeriales) dentro de modelos de más largo plazo que van más allá de la singularidad. ${ }^{4}$ La dimensión temporal y las variables de más larga duración tales como las características del Estado y los partidos, constituyen una buena puerta de entrada para comprender las similitudes y diferencias y abrir preguntas e hipótesis acerca de los modos de ejercicio del poder político.

El análisis acerca de la trayectoria de los grupos de poder, que con sus decisiones definen los destinos del país, encontró eco en un conjunto significativo de estudios del campo de las ciencias sociales. ${ }^{5}$ En Perú el tema ha sido objeto de análisis desde

\footnotetext{
${ }^{3}$ La base de datos elaborada por el Observatorio Electoral de América Latina (OBLAT) con sede en la Facultad de Ciencias Sociales de la UBA, se configuró a partir de datos públicos: páginas oficiales estatales, ministerios, currículums personales, redes sociales personales, medios periodísticos). Se toma como unidad de análisis a cada ministro o ministra y se consideraron las siguientes dimensiones: 1. Sociodemográficas (país, período, ministerio, puesto, sexo, lugar de nacimiento); 2. Socioeducativas: (carrera, universidad, posgrado, país de realización del posgrado, título de posgrado); 3. Ocupacionales: (trayectoria, ocupación en un puesto directivo, actividad de desempeñó, origen de la empresa, ocupación anterior al cargo, sector, actividad, origen de la empresa), 4. Participación política (participación en partidos, en organismos de la sociedad civil, en ONGs).

${ }^{4}$ Sobre la sociología y la posibilidad de presentar perspectivas híbridas véase: Tilly (1991), Dogan y Pahre (1993), Adams, Clemens y Orloff (2005). Una síntesis sobre estos debates puede leerse en Giordano (2011).

${ }^{5}$ Entre los trabajos pioneros podemos ciar a los clásicos de Wright Mills ([1956] 1963) y De Imaz ([1962] 1965). En Argentina hay un conjunto importante de trabajos sobre el tema. En particular destacamos los materiales producidos por el Observatorio de las élites argentinas, dirigido por Paula Canelo, Ana Castellani y Mariana Heredia en la Universidad Nacional
} 
Radiografía de los gabinetes ministeriales en Brasil y Perú (2016-2018). Un análisis comparativo

diferentes enfoques: los estudios centrados en una perspectiva socioeconómica respecto de las pujas de interés y los alineamientos entre las distintas fracciones de las clases dominantes (Boschi \& Diniz, 2004; Bresser Pereira, 2006, 2012, 2017; Bresser Pereira \& Diniz, 2009; Diniz, 1978, [1997] 2000; Furtado [1959] 1962, 1965, 1992, 1998; Martuscelli, 2013, 2018; Singer, 2009, 2012, 2015 para el caso de Brasil, y Malpica Santisteban, 1964; Thorp \& Zeballos, 2001; Vásquez, 2000; Durand, 2013, para el de Perú), los análisis acerca del problema de la captura del Estado, en particular en el caso de Perú (Távara, 2004; Durand, 2006; Crabtree \& Durand, 2017) y las investigaciones que intentan abordar la relación entre élites económicas, políticas y Estado (Brasil: Trindade, 1986; Cardoso, [1972] 1993; Codato \& Franz, 2017, 2018; Codato et. al., 2016, 2017; Perissinotto, et. al., 2015; D’Araujo, 2009, 2011, 2014 / Perú: Cotler, 1998; Cotler y Grompone, 2000; Degregori, 2001; Dargent, 2014, Durand, 2017).

Sin embargo, son pocos los análisis en clave comparativa latinoamericana y son también escasos los estudios que aborden una coyuntura reciente como la de esta investigación. ${ }^{6}$

\section{Los gabinetes de Pablo Kuczynski (2016-2018) y Michel Temer (2016-2018) en clave comparada}

Michel Temer (2016-2018), es un hombre que proviene del histórico Partido Movimento Democrático Brasileiro (PMDB) ${ }^{7} \mathrm{y}$, a diferencia de PPK, no fue electo para

\footnotetext{
de San Martín. En Brasil pueden ser mencionados las producciones del Observatório de elites políticas e sociais do Brasil de la Universidad Federal de Paraná (UFP) coordinado por Adriano Codato y de Maria Celina D'Araujo en el Centro de Pesquisa e Documentação de História Contemporânea do Brasil de la Fundação Getulio Vargas (Cpdoc/FGV).

${ }^{6}$ Para América Latina véase sumariamente: Viguera (1996), Flores Andrade (2003), Durand (2010), Rodríguez (2014), Vommaro (2015), Vommaro (2017), Castellani (2008, 2016, 2018), Ansaldi (2017), Canzani (2016), González Bozzolasco (2018), Giordano (2014), Soler (2018).

${ }^{7}$ El PMDB es uno de los partidos más antiguos del sistema político brasileño que se formó en la coyuntura de la distensión de la dictadura militar. Según Salas Oroño (2016), la fisonomía del PMDB se configuró durante los años de Fernando Henrique Cardoso (1995-1998/1999-2002), donde estableció un vínculo "de coalición” colocando cargos en el Ejecutivo y en los esquemas de poder regionales y municipales a cambio de cohesionar una serie de recursos políticos parlamentarios que votaran a favor del oficialismo. Durante el gobierno de Lula, cuando se destapó el llamado "escándalo del mensalão" alrededor del año 2005, el PT decidió establecer contactos orgánicos con el PMDB para asegurar, entre otras cosas, que no
} 
ejercer el cargo de la presidencia. En 2014 fue candidato a vicepresidente e integró la fórmula presidencial que llevó al poder por segunda vez a Dilma Rousseff (Partido dos Trabalhadores-PT). La fórmula alcanzó una victoria muy ajustada $(51,64 \%)$ frente al candidato Aécio Neves (Partido da Social Democracia Brasileira-PSDB). Por causa de ese triunfo limitado el PT debió sortear dificultades políticas en un marco de acuerdos débiles con distintos aliados (entre ellos el partido de Temer). Además, hubo un asedio sistemático del poder judicial a través de las denuncias contra el expresidente Luiz Inácio Lula da Silva y contra Rousseff, esta última en el marco de la causa por las denominadas pedaladas fiscales. ${ }^{8}$ Este proceso concluyó con el pedido de impeachment contra Rousseff y su posterior destitución. El partido de Temer, quien todavía ejercía como vicepresidente, abandonó la coalición mantenida con el partido de gobierno y apoyó el proceso destituyente. Así, Temer accedió a la presidencia con un programa de gobierno que, como hemos señalado más arriba, asumió las reivindicaciones de las grandes corporaciones y rompió con las políticas de gobierno impulsadas por los gobiernos petistas. Tras asumir, Temer conformó un gabinete con mayoría de hombres blancos y fue el primero en reducir significativamente la participación de mujeres (sólo hubo dos en toda la gestión) y en excluir a las minorías étnicas desde la transición democrática en 1985.

Pedro Pablo Kuckzynski ganó las elecciones en 2016, luego de imponerse en segunda vuelta frente a Keiko Fujimori, la hija del Alberto Fujimori (1990-2000). Kuczynski se había postulado por primera vez como candidato a presidente en el año 2011, sin embargo, optó por presentarse con un nuevo partido político denominado Peruanos por el Kambio (PPK-2014), cuyas iniciales referencian su nombre (sobre las elecciones véase Tuesta Soldevilla, 2016). Aun cuando tenía una larga trayectoria en el

prosperaran los pedidos de impeachment y a la vez garantizar acuerdos territoriales y regionales. Formalmente el PMDB ingresó a la coalición de gobierno en 2010, en la elección donde Dilma Rousseff llegó por primera vez a la presidencia.

${ }^{8}$ La pedalada fiscal suponía el adelanto de fondos públicos para cubrir programas de responsabilidad del gobierno. 
Radiografía de los gabinetes ministeriales en Brasil y Perú (2016-2018). Un análisis comparativo

plano público y privado, Kuczynski era el candidato que expresaba a la derecha liberal, con un perfil empresarial acuñado a lo largo de su trayectoria laboral: integró el directorio de varias empresas vinculadas al sector minero y energético y trabajó en organismos multilaterales como el Banco Mundial. También poseía trayectoria política previa, pues había sido ministro de Energía y Minas durante el gobierno de Belaúnde Terry (1980-1985) y ministro de Economía y Primer ministro del gobierno de Alejandro Toledo (2001-2006). En este último cargo estableció vínculos con parte de los funcionarios y las funcionarias quienes luego integraron su propio gobierno. El gobierno de PPK fue de inestabilidad política motivada fundamentalmente por la imposibilidad de construir consensos en una cámara con mayoría absoluta del fujimorismo, las dificultades para establecer un rumbo económico y político y las denuncias de corrupción. Por tal motivo, se realizaron tres cambios de gabinetes. El primero estuvo conformado por una mayoría de figuras del ámbito tecnócrata y empresarial de procedencia de Lima, la capital política, económica y financiera del país y en los otros dos intentó compensar ese perfil con la incorporación de un funcionariado que provenía del mundo de la política. La experiencia de gobierno de PPK duró muy poco, y en el marco de denuncias cruzadas de corrupción y de conflicto de interés donde quedaban involucrados funcionarios y funcionarias, Kuckysnki presentó su renuncia, la cual se aprobó en el Congreso el 23 de marzo de 2018.

\section{Perfil social, educativo y político}

El gabinete brasileño estaba compuesto por 29 ministerios y secretarías con rango ministerial y en total hubo 66 funcionarios y funcionarias que pasaron por esos cargos. El de Pedro Pablo Kuczynski estaba conformado por 19 carteras y, debido a la rotación y cambios ministeriales, hubo 45 ministros y ministras que pasaron por su gestión. En cuanto al perfil sociodemográfico, la edad promedio del gabinete brasileño era de 54 años y, a diferencia de Perú, resalta la preponderancia de hombres, pues 
solamente hubo 2 (3\%) mujeres en todo el gabinete. La escasa presencia femenina continúa una tendencia histórica que alcanzó apenas a ser muy parcialmente revertida a partir de los mandatos de Lula (D'Araujo, 2009). El gabinete peruano presenta las siguientes características: la edad promedio es de 55 años y, si bien hay una preeminencia de varones, 14 (31\%) cargos estuvieron ocupados por mujeres, por lo cual se convirtió en el gabinete con más mujeres de la historia del país.

En cuanto a la procedencia del funcionariado, en Brasil los miembros del gabinete se distribuyeron del siguiente modo: la región Sudeste del país, (Minas Gerais, Rio de Janeiro y São Paulo) con 36\%, seguida por la región Sur (Paraná, Santa Catarina y Rio Grande do Sul) con 20\% y el Noreste (Bahía y Pernambuco) con el 18\%. Esta distribución geográfica encuentra relación con la mayor diversificación del sistema productivo y el regionalismo del país, aunque históricamente ha prevalecido en términos económicos y políticos la región Sudeste. Asimismo, contrasta con el caso peruano donde hubo una importante concentración de ministros y ministras provenientes del departamento de Lima (67\%) mientras el 33\% restante pertenecían a los departamentos del interior. Históricamente el país tuvo una división geográfica muy pronunciada entre los departamentos ubicados en la costa (los más ricos), la sierra (en segundo lugar) y la selva (la más desfavorecida) y, en los últimos tiempos, la tendencia a la concentración del poder en el área Lima fue más evidente.

Con respecto al nivel educativo, el conjunto de ministros y ministras de ambos países posee una carrera universitaria, exceptuando un único caso en el gabinete brasileño. En Brasil la formación universitaria se repartió del siguiente modo: en primer lugar, las Universidades públicas, luego las privadas laicas y, mucho más atrás, las privadas confesionales y la formación en el exterior. En Perú, en cambio, la mayoría obtuvo formación en el ámbito de la educación privada, en primer lugar, en Universidades confesionales y luego en establecimientos laicos (ver Cuadro 1). 
Radiografía de los gabinetes ministeriales en Brasil y Perú (2016-2018). Un análisis comparativo

\section{Cuadro 1. Formación universitaria}

\begin{tabular}{|c|c|c|c|c|c|c|}
\hline \multirow[b]{2}{*}{ Pública } & \multicolumn{2}{|c|}{ Brasil } & \multicolumn{2}{|c|}{ Perú } & \multicolumn{2}{|c|}{ Total general } \\
\hline & 31 & $47,0 \%$ & 12 & $26,7 \%$ & 43 & $38,7 \%$ \\
\hline Privada laica & 23 & $34,8 \%$ & 12 & $26,7 \%$ & 35 & $31,5 \%$ \\
\hline $\begin{array}{l}\text { Privada } \\
\text { confesional }\end{array}$ & 8 & $12,1 \%$ & 21 & $46,7 \%$ & 29 & $26,1 \%$ \\
\hline Extranjero & 3 & $4,5 \%$ & & & 3 & $2,7 \%$ \\
\hline No realizó & 1 & $1,5 \%$ & & & 1 & $0,9 \%$ \\
\hline Total general & 66 & $100 \%$ & 45 & $100 \%$ & 111 & $100 \%$ \\
\hline
\end{tabular}

Fuente: elaboración propia en base a datos del Observatorio Electoral de América Latina, OBLAT.

Las carreras de grado donde tuvieron formación los funcionarios y las funcionarias encuentran relación con el perfil del gobierno. Más arriba señalamos una diferencia crucial entre Temer y PPK en cuanto al perfil del funcionariado. Mientras en Brasil hay una clase política histórica, que tomó como bandera el programa de los principales grupos económicos, en Perú el presidente y los principales miembros del gabinete provienen del mundo empresarial o poseen un rasgo tecnocrático. Por este motivo resulta notorio que la carrera predominante en Brasil es la de abogacía, una disciplina tradicionalmente relacionada con la formación política (D’Araujo, 2009), mientras en Perú es la de economía, históricamente vinculada con el mundo de los negocios. Si observan los datos con cuidado, la diferencia es notable: en Brasil un 46\% se formó en el campo de las ciencias jurídicas, seguido por ingeniería (12\%), economía (11\%) y administración de empresas (11\%), entre otras; mientras que en Perú un 33\% del total alcanzó su formación en la carrera de economía, por encima de abogacía (18\%), ingeniería (11\%), y otras (ver en Anexos, cuadro 2).

Sin embargo, un elemento mediante el cual es posible delinear el perfil de gobierno con mayor precisión es la formación en el nivel de posgrado (ver Gráfico 1). En países como Argentina, Brasil y México, donde las Universidades públicas suelen gozar de mayor prestigio y calidad educativa, la impronta de los profesionales termina 
de configurarse en la formación de posgrado, donde pareciera haber mayores posibilidades de escoger un perfil de formación "a medida". ${ }^{9}$ Los datos nos muestran lo siguiente: en Brasil el número de funcionarios y funcionarias con título de posgrado es de $68 \%$, mientras en Perú es mayor y asciende a un $91 \%$ del total del gabinete. Es más significativa la diferencia respecto de la institución en la cual han realizado sus posgrados. En Brasil el 44\% realizó sus posgrados en el país, 17\% en Estados Unidos, $6 \%$ en Europa y $2 \%$ en otros lugares (el $32 \%$ restante no realizó ningún posgrado). En cambio, en Perú la relación aparece invertida, es mayor el número de quienes optaron por formarse en el exterior: la distribución de casos mostró que el $31 \%$ se formó en Estados Unidos, $27 \%$ en el país de origen, 20\% en América Latina, 11\% en Europa, y $2 \%$ en otros lugares (el 9\% restante no realizó ningún posgrado). Este rasgo vuelve a ilustrar un elemento ya señalado. La formación en Universidades públicas y nacionales es una característica de larga data en Brasil, así como también la formación de cuadros para la administración pública. Según un investigador brasileño especializado en temas de élites y clases dominantes, "las universidades forman elites [...] porque está vinculado eso con la estructura organizativa del Estado que es fuerte, muy fuerte [la] presencia de ideología meritocrática" (Daniel, 23 noviembre de 2018).

En Perú no hay una tradición de fortaleza de las Universidades públicas, por lo cual hay una tendencia a optar por la formación privada, de la misma manera que en el nivel de los posgrados, donde la tendencia ha sido la de una formación en el exterior y, en particular, en los Estados Unidos.

\section{Gráfico 1. Formación de postgrado}

\footnotetext{
${ }^{9}$ Un dato que fortalece esta idea es el siguiente: de los ocho casos estudiados en el proyecto general, Argentina, Brasil y México son los países que evidencian mayor formación de ministros y ministras en Universidades públicas, a diferencia de Chile, Colombia, Paraguay, Perú.
} 
Radiografía de los gabinetes ministeriales en Brasil y Perú (2016-2018). Un análisis comparativo

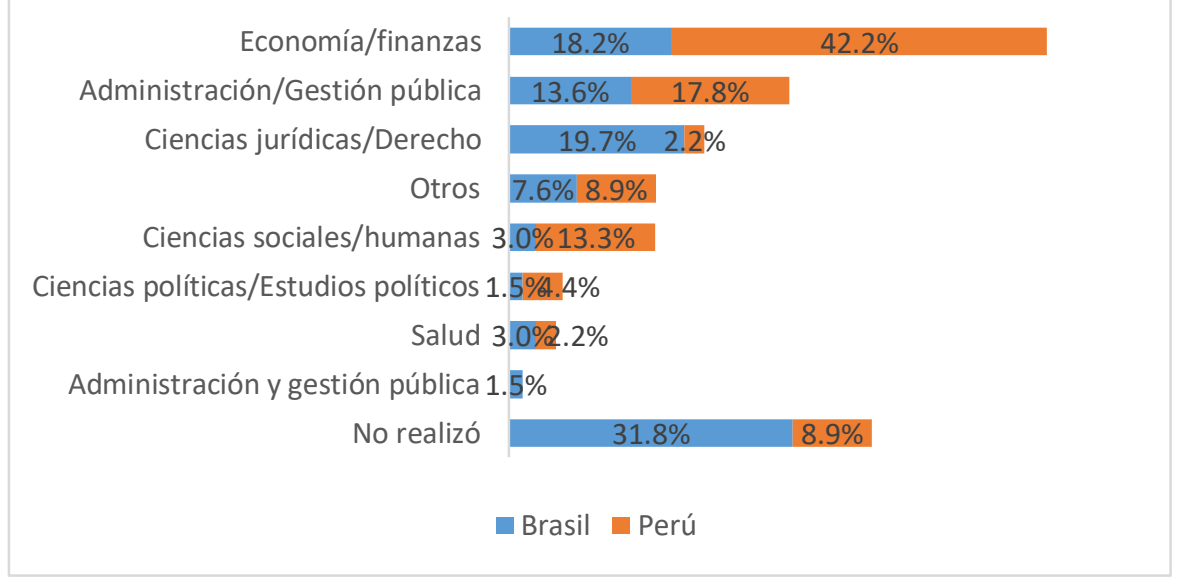

Fuente: elaboración propia en base a datos del Observatorio Electoral de América Latina, OBLAT.

En cuanto al tipo de postgrado, nuevamente en Brasil hay un mayor número de quienes eligen una formación vinculada al mundo de las ciencias jurídicas y el derecho (19,7 \%), y luego la economía y las finanzas (18\%); mientras en Perú el mayor porcentaje está concentrado entre quienes realizaron una formación en economía y finanzas (42\%), seguido de administración/gestión pública (18\%).

Otra de las dimensiones analizadas es la participación en partidos políticos de los funcionarios (ver Cuadro 3). En congruencia con el perfil ministerial ilustrado para el caso de Brasil, el número de quienes se reconocen como miembros de un partido asciende a $65 \% .{ }^{10}$ La presente composición del gabinete brasileño se revela coincidente con algunas tendencias históricas: altos grados de involucramiento en la política y la gestión pública en cargos directivos como instancia previa al arribo al cargo ministerial; y preponderante filiación de los funcionarios al partido del cual proviene el presidente.

\footnotetext{
${ }^{10}$ Para construir estos datos se tomó la siguiente definición: sólo se consideraron partidarios a quienes tuvieran una posición pública de pertenencia a una determinada fuerza política reconocida en medios periodísticos, fuentes oficiales, currículos, etc. Es decir que, los números presentados, eventualmente, podrían llegar a ser algo mayor, en el caso de que hubiera algún funcionario o funcionaria que no hiciera pública su inscripción política.
} 
Este último rasgo, se acentuó durante los gobiernos del PT, por tratarse de una fuerza política que llegaba al poder estatal por primera vez (Codato \& Franz, 2017). Según lo revelado por los entrevistados, en Brasil hay mucha estabilidad para los sectores de la burocracia más fijos en el Estado. Si bien cada nuevo gobierno introduce cambios y designa personal de su confianza, hay una manutención de un patrón de funcionamiento de la burocracia, de ahí que suele conformarse cierta élite o casta.

En el caso de Perú, por el contrario, coexisten empresarios y empresarias, tecnócratas, políticos y políticas y son muy pocos quienes se reconocen partidarios de una fuerza política. Inclusive en una de las entrevistas realizada a un alto funcionario que desempeñó en el área de seguridad durante el gobierno de PPK sostuvo, en referencia a un colega: "jamás se le cruzaría por la mente entrar a un partido. Se suicida antes. Igual que yo, igual que el $95 \%$ de los peruanos. Nos suicidamos antes de entrar [...] asusta cuando ves que un ministerio está manejado por un partido político" (Julio, 10 de mayo de 2018). Esta expresión es coincidente con el perfil general de los cargos del gabinete: sólo un $31 \%$ de los cargos pertenecen a hombres o mujeres con participación política partidaria.

Cuadro 3. Participación política partidaria

\begin{tabular}{|c|c|c|c|c|}
\hline \multirow[b]{2}{*}{ PMDB } & \multicolumn{2}{|c|}{ Brasil } & \multicolumn{2}{|c|}{ Perú } \\
\hline & 18 & $41,9 \%$ & & \\
\hline PSDB & 6 & $14,0 \%$ & & \\
\hline PP & 4 & $9,3 \%$ & & \\
\hline PPS & 4 & $9,3 \%$ & & \\
\hline PRB & 2 & $4,7 \%$ & & \\
\hline РTB & 2 & $4,7 \%$ & & \\
\hline PV & 2 & $4,7 \%$ & & \\
\hline Otros & 5 & $11,6 \%$ & & \\
\hline $\begin{array}{l}\text { Peruanos Por el } \\
\text { Kambio }\end{array}$ & & & 11 & $78,6 \%$ \\
\hline APRA & & & 2 & $14,3 \%$ \\
\hline
\end{tabular}


Radiografía de los gabinetes ministeriales en Brasil y Perú (2016-2018). Un análisis comparativo

\begin{tabular}{|llccc|}
$\begin{array}{l}\text { Partido Popular } \\
\text { Cristiano }\end{array}$ & & & & \\
\hline Si & $\mathbf{4 3}$ & $\begin{array}{c}\mathbf{6 5 , 2 \%} \\
(\mathbf{1 0 0 \%})\end{array}$ & $\mathbf{1 4}$ & $\begin{array}{c}\mathbf{3 1 , 1 \%} \\
\mathbf{( 1 0 0 \%}\end{array}$ \\
\hline No & $\mathbf{2 3}$ & $\mathbf{3 4 , 8 \%}$ & $\mathbf{3 1}$ & $\mathbf{6 9 \%}$ \\
\hline Total general & $\mathbf{6 6}$ & $\mathbf{1 0 0 \%}$ & $\mathbf{4 5}$ & $\mathbf{1 0 0 \%}$ \\
\hline
\end{tabular}

Fuente: elaboración propia en base a datos del Observatorio Electoral de América Latina, OBLAT.

Asimismo, la participación en organismos de la sociedad civil muestra la siguiente evidencia: en Brasil un 13,9 \% (9 casos) formó parte de organismos corporativos empresariales, en tanto en Perú el número asciende a un 27\% (12 casos). Si bien la cantidad de ministros o ministras no es demasiado alta con relación a Brasil, mirado en términos proporcionales se confirma la tendencia de una mayor presencia de funcionariado vinculado al mundo empresarial (ver Cuadro 4).

\section{Cuadro 4. Participación en organismos de la sociedad civil}

\begin{tabular}{|lc|c|c|c|c|c|}
\hline & \multicolumn{2}{c}{ Brasil } & \multicolumn{2}{c|}{ Perú } & & \\
\cline { 2 - 7 } Corporativos & & & & & & \\
(empresariales) & 9 & $13,6 \%$ & 12 & $26,7 \%$ & 21 & $18,9 \%$ \\
Gremiales/Sindicales & 5 & $7,6 \%$ & 1 & $2,2 \%$ & 6 & $5,4 \%$ \\
Religiosos & 6 & $9,1 \%$ & & & 6 & $5,4 \%$ \\
Deportivos/Clubes & & $0,0 \%$ & 2 & $4,4 \%$ & 2 & $1,8 \%$ \\
Otros & 1 & $1,5 \%$ & & & 1 & $0,9 \%$ \\
No registra & 45 & $68,2 \%$ & 30 & $66,7 \%$ & 74 & $66,7 \%$ \\
Total general & 66 & $100 \%$ & 45 & $100 \%$ & 111 & $100 \%$ \\
\hline
\end{tabular}

Fuente: elaboración propia en base a datos del Observatorio Electoral de América Latina, OBLAT.

\section{Trayectoria ocupacional}

La trayectoria ocupacional constituye otro indicador relevante para caracterizar el perfil de los gabinetes ministeriales. Para ello tomamos dos dimensiones: el desempeño profesional a lo largo de toda la carrera, así como también, el último cargo antes de asumir la función de ministro o ministra. El análisis a partir de esta doble 
entrada nos permitió caracterizar con mayor fidelidad los recorridos y las biografías diversas. Así, fue posible identificar tres tipos de trayectorias: pública, público-privada y privada. La mayoría de quienes poseen una trayectoria realizada íntegramente en la órbita pública son cuadros políticos que desempeñaron cargos electivos o ejecutivos en la órbita estatal o bien desarrollaron una trayectoria ligada a la gestión pública mediante el ejercicio profesional. El funcionariado que posee una trayectoria público-privada, presenta dos tipos de perfil: aquellos que transitaron varios años en el mundo privado y luego hicieron un salto hacia la vida política en cargos electivos o en puestos dentro de la órbita ejecutiva (secretarios, subsecretarios), o bien, quienes alternan entre el mundo privado y el público (un funcionario o funcionaria cuya trayectoria tiene base en el sector privado y se incorpora intermitentemente a la gestión pública, a partir de ser convocado o convocada por el gobierno). ${ }^{11}$ La tercera trayectoria refiere a quienes provienen íntegramente del mundo privado: en general se trata de empresarios o CEOs, directores o gerentes del mundo empresarial o quienes poseen consultoras profesionales vinculadas al mundo de los negocios. En algunos pocos casos, están quienes tienen trayectoria en el mundo privado pero no provienen del mundo empresarial sino del ámbito artístico o periodístico.

El análisis de la trayectoria ocupacional muestra datos ilustrativos. En Brasil el $61 \%$ del funcionariado trabajó en la órbita pública en forma exclusiva, el 38\% posee una trayectoria público-privada y sólo el 2\% (un sólo miembro) tuvo una trayectoria en el ámbito privado exclusivamente. En Perú la relación es inversa: el 69\% del gabinete ministerial tiene una trayectoria público-privada, mientras que $16 \%$ tuvo un recorrido exclusivo en el mundo privado y $16 \%$ una trayectoria pública pura (ver Cuadro 5).

\footnotetext{
${ }^{11}$ En esta última situación es común el fenómeno de la "puerta giratoria" cuyo problema ha sido un rasgo de Perú incluso antes del gobierno de PPK (véase Durand, 2017).
} 
Radiografía de los gabinetes ministeriales en Brasil y Perú (2016-2018). Un análisis comparativo

\section{Cuadro 5. Trayectoria ocupacional}

\begin{tabular}{|lc|c|c|c|c|c|}
\hline & \multicolumn{2}{c}{ Brasil } & \multicolumn{2}{c|}{ Perú } & \multicolumn{2}{c|}{ Total general } \\
\cline { 2 - 7 } Público-privada & 25 & $37,9 \%$ & 31 & $68,9 \%$ & 56 & $50,5 \%$ \\
Pública pura & 40 & $60,6 \%$ & 7 & $15,6 \%$ & 47 & $42,3 \%$ \\
Privada pura & 1 & $1,5 \%$ & 7 & $15,6 \%$ & 8 & $7,2 \%$ \\
Total general & 66 & $100 \%$ & 45 & $100 \%$ & 111 & $100 \%$ \\
\hline
\end{tabular}

Fuente: elaboración propia en base a datos del Observatorio Electoral de América Latina, OBLAT.

Entre quienes registran un paso por el mundo privado (es decir quienes tienen trayectoria privada pura o público-privada), otra de las dimensiones relevadas refiere a la ocupación de cargos directivos o la propiedad de capital, bajo la forma de activos fijos o financieros. Dentro del gabinete de Temer, el número de funcionarios que tuvieron cargos directivos o fueron propietarios de empresas es de 20 (30\%). Todos ellos, con excepción de un solo ministro, el cual trabajó durante gran parte de su trayectoria en empresas multinacionales (entre ellas Mercedes Benz y British Petroleum), lo hicieron en empresas dentro del ámbito nacional.

En cambio, en el gabinete de PPK, dentro del conjunto de funcionarios y funcionarias que tuvieron actividades en el sector privado, 30 (67\%) se desempeñaron en puestos directivos o fueron propietarios a lo largo de su trayectoria, de éstos, 12 (27\%) lo hicieron mayoritariamente en firmas extranjeras.

Asimismo, con relación a la participación en organismos multilaterales de crédito en algún momento de la trayectoria, la tendencia se repite: en Brasil, solamente el presidente del Banco Central presenta experiencia laboral en el Fondo Monetario Internacional (FMI), mientras en Perú hay una mayor extranjerización en la formación e ideario de los cuadros ministeriales: hay 11 casos de participación en organismos multilaterales, 7 en el Banco Interamericano de Desarrollo (BID) y 4 en el Banco Mundial (BM). 
En cuanto al último cargo desempeñado antes de asumir como ministro o ministra, los números arrojan los siguientes datos que se observan en el Cuadro 6.

\section{Cuadro 6. Ocupación anterior al cargo}

\begin{tabular}{|lc|c|c|c|c|c|}
\hline & \multicolumn{2}{c}{ Brasil } & \multicolumn{2}{c|}{ Perú } & \multicolumn{2}{c|}{ Total general } \\
\cline { 2 - 7 } & & & & & & \\
Público & 58 & $87,9 \%$ & 26 & $57,8 \%$ & 84 & $75,7 \%$ \\
Privado & 8 & $12,1 \%$ & 16 & $35,6 \%$ & 24 & $21,6 \%$ \\
Tercer sector & & & 3 & $6,7 \%$ & 3 & $2,7 \%$ \\
Total general & 66 & $100 \%$ & 45 & $100 \%$ & 111 & $100 \%$ \\
\hline
\end{tabular}

Fuente: elaboración propia en base a datos del Observatorio Electoral de América Latina, OBLAT.

En Brasil solamente el 12\% tenía funciones en el mundo privado al momento de ser convocado a integrar el ministerio y un $88 \%$ lo hacía en la órbita pública. En Perú los números son significativamente diferentes: el 36\% trabajaba en el mundo privado y el 58\% lo hacía en el sector público (ver cuadro 7 en Anexos).

En ambos países, entre quienes trabajaban en la órbita pública, alrededor del $50 \%$ lo hacía en algún cargo ejecutivo (en la mayoría de los casos en un rango inferior, o en una escala subnacional). Sólo en el caso de Perú hay funcionarios que trabajaron en el tercer sector antes de asumir el cargo ministerial (3 casos).

En ambos países se resalta la procedencia mayoritaria, en lo que respecta al sector privado, en su desempeño en el área de la asesoría profesional a empresas (jurídica, contable, en publicidad e investigación de mercados de consultoría y en gestión en general) y, en segundo término, del sector bancario y financiero. Bastante por detrás se ubican el sector productivo y de telecomunicaciones y consumo culturales.

Respecto del sector público, se destaca el número de quienes se desempeñaron en el poder ejecutivo, generalmente en cargos de menor jerarquía (secretarios, subsecretarios y directores de entes autárquicos dentro de la órbita estatal) o en niveles subnacionales de gobierno. En Brasil, no obstante, el paso por la instancia legislativa es considerablemente mayor al que registra Perú. Esto se debe a que en el país brasileño 
Radiografía de los gabinetes ministeriales en Brasil y Perú (2016-2018). Un análisis comparativo

el ámbito legislativo suele ser el que permite un "salto" en la trayectoria del funcionariado hacia el área ejecutiva o, a la inversa, el lugar de retorno tras haberse desempeñado en el ejecutivo.

\section{Reflexiones desde la sociología histórica}

En este análisis donde combinamos la sociología histórica y la sociología política, la pregunta por el cuándo resulta crucial. El sociólogo Francisco Durand (2010) sostiene que el neoliberalismo de los noventa facilitó la llegada al poder de los empresarios a la política, a partir de la profunda modificación en la estructura del poder económico. ${ }^{12}$ En Brasil Fernando Collor de Mello (1990-1992), fue el primer presidente con esas características del país y el gobierno de Fernando Henrique Cardoso (19951998/1999-2002), del Partido da Social Democracia Brasileira (PSDB), quien introdujo el modelo de ajuste estructural de corte neoliberal ya como Ministro de Hacienda durante el período de Itamar Franco (1992-1994), trajo un perfil tecnocrático (D’Araujo, 2009; Codato \& Franz, 2017). Durante los gobiernos del PT (2003-2016) hubo una mayor preponderancia de un perfil político y militante: sindicalistas, minorías (étnicas) y mujeres (D’Araujo, 2009; Codato \& Franz, 2017). Tras la destitución de Rousseff, el gobierno de Temer retomó la senda de la conformación de gabinetes con impronta de políticos y tecnócratas. En una de las entrevistas realizada para esta investigación, un ex Ministro del área de Ciencia y Tecnología durante los gobiernos del PT planteó lo siguiente: "es una cuestión de un país capitalista. El empresario

\footnotetext{
${ }^{12} \mathrm{El}$ autor sostiene que en América Latina hubo cinto tipos de acción empresarial: 1. protestas y revueltas empresariales; 2. golpes de Estado; 3. invasiones militares; 4. elecciones con acomodos empresariales a partidos o candidaturas populistas, y 5 . candidaturas y gobiernos conservadores de empresarios. Los tres primeros tipos son más bien excepcionales y provocan distintos niveles de violencia; los dos últimos predominan y son pacíficos. En esos años hubo tres presidentes que pertenecían a la órbita empresarial: Gonzalo Sánchez de Losada (1993-1997/2002-2003), un empresario de la minería en Bolivia, Fernando Collor de Mello (1990-1992), un hombre vinculado a los medios de comunicación en Brasil y Juan Carlos Wasmosy (1993-1998) un empresario de la construcción en Paraguay.
} 
participa siempre en la política. $\mathrm{O}$ bien participan directamente $[\ldots]$ o bien participan a través de sus apoderados y sus representantes. No altera el juego de la política" (Claudio,15 de julio de 2018).

En Perú la década de 1990 también constituyó un punto de inflexión. Durante el gobierno autoritario de Fujimori (1990-2000), se incorporó el empresariado en funciones ministeriales y una de las figuras emblemáticas fue el economista Carlos Boloña. A diferencia de Brasil, donde los gobiernos del PT introdujeron una disputa hegemónica por el modelo de país y la puja distributiva, en Perú, desde los noventa hay una continuidad y cierto acuerdo en los grandes parámetros de signo neoliberal (sobre la economía durante los noventa véase Vásquez, 2000; Thorp y Zevallos, 2001). El modelo económico, con base a la producción de recursos primarios, se continuó durante los gobiernos de Alejandro Toledo (2001-2006), Alan García (2006-2011) y Ollanta Humala (2011-2016), aunque con una mayor inversión en el área social durante este último. Desde entonces, como planteó el conjunto de los entrevistados de Perú, los gobiernos que siguieron, con excepción del gobierno de Alan García (2006-2011), tuvieron una fuerte presencia de tecnócratas, especialmente en carteras muy sensibles para el país (como Minería y Economía) (véase Durand \& Crabtree, 2017).

A diferencia del caso brasileño, donde las experiencias del PT marcaron transformaciones en cuanto al modelo socioeconómico y en las élites políticas, en Perú la nota fue de continuidad. Un alto funcionario peruano del gobierno de PPK sostuvo al respecto: "hay un consenso básico. Tenemos acuerdo de libre comercio con todo el mundo. Pertenecemos a la Alianza para el Pacífico [...] Hay un consenso básico y creo que eso se ha mantenido en los gabinetes" (Julio, 10 de mayo 2018). Aun cuando es posible hallar líneas de continuidad, especialmente en algunas carteras como ya hemos mencionado, hay un acuerdo generalizado en que el gobierno de PPK le imprimió un perfil más orientado hacia los tecnócratas y empresarios, es decir, un perfil de gabinete con visión empresarial, como también señalaron varias de las entrevistas realizadas. 
Radiografía de los gabinetes ministeriales en Brasil y Perú (2016-2018). Un análisis comparativo

Estos rasgos de los gabinetes ministeriales de los gobiernos de Temer y PPK se entrelazan con otros elementos de larga duración propios del análisis sociológico histórico. En Brasil hay una formación temprana del Estado (Ansaldi y Giordano, 2012), aunque la expansión de la arena política ocurrió de manera lenta y restringida (Trindade, 1986). Por ello es posible señalar que la estatalidad brasileña posee mayor preponderancia por sobre los partidos y los movimientos populares, en la definición de las cuestiones centrales de la política (De Riz, 1986). En cuanto a los partidos, éstos han sido de creación tardía y con rasgos de debilidad y fragmentación (Ansaldi, 1995). La tendencia a la primacía de los grupos políticos de carácter regional dificultó la posibilidad de construir partidos orgánicos de alcance nacional. Y, con excepción del Partido Comunista, creado en 1922, al día de la fecha no existe ningún partido cuya creación se remonte a un momento anterior al golpe de Estado de 1964 (Meneguello, 2002; Mainwaring, [1995] 1996; Ansaldi, 1995).Si bien el de Brasil suele ser ubicado como un caso de debilidad partidaria en el conjunto de la literatura, en lo que respecta a la administración del poder, las élites políticas en el Estado han ocupado un lugar de mediación respecto de los intereses de los distintos actores de la sociedad. Es por ello que, aun cuando hubo un gobierno con una fuerte orientación hacia el mercado, las élites económicas vehiculizaron sus demandas a través de las históricas élites políticas. En Brasil la presencia de empresarios y empresarias es difusa y tienen un perfil más técnico o muy vinculado al partido. Como sostuvo un investigador brasileño, podría decirse que hay una regla de oro "que es poner gente que son de los partidos para componer el gobierno [...] la idea de lobby demuestra claramente cómo se articulan esos intereses empresariales privados con el Estado" (Daniel, 23 de noviembre de 2018). El empresariado no tiene una necesidad tan imperiosa de colocar personalidades del mundo empresarial, sino más bien construir el lobby y los vehículos políticos para satisfacer sus propios intereses. 
Además de los factores mencionados pueden señalarse otros dos rasgos en el caso de brasileño. El presidencialismo de coalición promueve la realización de acuerdos partidarios entre las fuerzas. En ese marco, el presidente electo debe atraer a otros partidos para integrar el gobierno conformando un esquema de acuerdo con distintas fuerzas políticas. Al mismo tiempo, la presencia de las bancadas corporativas en el parlamento favorece la participación del empresariado o de representantes de sus intereses en ese cuerpo parlamentario, canalizando sus demandas a través de la instancia legislativa. Consultado para esta investigación un ex Ministro brasileño del área de Cultura durante los gobiernos del PT sostuvo lo siguiente: "La composición del parlamento ya es bastante hegemónica de empresarios. Empresarios, hacendados y de las elites en general, o cuando no son de origen, defienden sus intereses" y, frente a la pregunta acerca del gabinete ministerial de Temer, indicó "los empresarios pudieron ir para la playa porque había quién estuviera defendiendo sus intereses" (Franco, 9 de noviembre de 2018).

Perú es un caso de formación estatal débil y tardía (Ansaldi y Giordano, 2012) y posee una histórica carencia de cuadros dirigenciales con trayectoria estatal (Cotler [1978] 2005). Además, tiene un sistema de partidos políticos débiles y fragmentados, con altos niveles de volatilidad -posiblemente los más altos de la región (véase sobre el tema Cotler, 1995; Tanaka, 2005, 2010; Mainwaring, 2009; Meléndez, 2012; Rubio, 2016). La conformación de un sistema de partidos moderno fue tardía, a diferencia de lo ocurrido en otros países andinos, en un territorio con una significativa fragmentación geográfica con dificultades para construir partidos nacionales robustos. Esta conjunción de elementos, sumado a una baja credibilidad de la democracia y las instituciones (Tanaka, 2010), contribuyó con la existencia de una preponderancia de los poderes fácticos en los procesos de definición de la política. Consultada para esta investigación una reconocida periodista peruana especializada en temas políticos en diversos medios de comunicación, sostuvo que durante la década de los noventa con el gobierno de 
Radiografía de los gabinetes ministeriales en Brasil y Perú (2016-2018). Un análisis comparativo

Fujimori se produjo el ingreso de los primeros empresarios en el Estado, cuya figura emblemática fue el Ministro de Economía y Finanzas Carlos Boloña (Clara, 11 de mayo de 2018). Desde entonces -agrega- hubo una combinación de tecnócratas y empresarios. Con relación al gobierno de PPK, la mayoría de las entrevistas sostuvo que más que empresarios, hubo un conjunto de "técnicos" o "independientes" con algunas figuras provenientes del mundo empresarial, pero el factor común fue la "visión empresarial de las cosas" (Julio, 10 de mayo 2018).

\section{Breves reflexiones finales}

Esta investigación es parte de un proyecto más amplio que compara las trayectorias de funcionarios y funcionarias de gobiernos con perfil empresarial en América Latina, desde el año 2000 hasta la actualidad. En este artículo tomamos dos países ubicados en los puntos más extremos de la comparación: Brasil es un caso de un gabinete con perfil político y Perú de un cuerpo ministerial con sesgo tecnocráticoempresarial. Como se señaló a lo largo del trabajo, el perfil de la máxima conducción de gobierno era distinto, Temer provenía del mundo de la política en cambio PPK, sin desmedro de su participación política previa, configuró un perfil con una fuerte impronta empresarial.

Con relación a la dimensión educativa, en Brasil hay mayor cantidad de funcionarios y funcionarias con carrera de grado y posgrado vinculada a las ciencias jurídicas, en tanto Perú presenta más cantidad de casos con una carrera desarrollada en el ámbito de la economía y la administración. La trayectoria ocupacional confirma la tendencia: en el país brasileño el número de quienes tuvieron una trayectoria pública y la cantidad de directivos de empresas es sensiblemente menor al caso de Perú, de igual modo que la participación en organismos multilaterales de crédito donde Brasil presenta menos casos que Perú. Este elemento muestra una mayor extranjerización de la economía y vinculación dependiente de los flujos de capital externo en el caso de Perú. 
Como hemos mencionado a lo largo del trabajo, una explicación analítica de largo aliento resulta crucial para comprender las diferencias entre los casos nacionales. En Brasil hay una formación temprana del Estado y una fuerte estatalidad por sobre los partidos para la definición de la política. Este rasgo se observa en el importante número de cuadros políticos con formación y trayectoria en el ámbito público. Perú es, por el contrario, un caso de formación estatal débil y tardía y posee una histórica carencia de cuadros dirigenciales para la ocupación de sus instituciones. Además, tiene un sistema de partidos políticos débiles y fragmentados. Esta conjunción de elementos contribuyó con la preponderancia de los poderes fácticos en los procesos de definición de la política y el gobierno a lo largo de la historia, potenciado por el perfil y la visión empresarial que le pretendió impulsar el gobierno de PPK.

A partir del análisis comparativo, este artículo ha buscado ofrecer algunas claves para pensar la trayectoria de los funcionarios y funcionarias estatales en dos países de América Latina, con el objetivo de abrir preguntas acerca de los distintos modos del ejercicio del poder político en una clave de análisis latinoamericana.

\section{Referencias bibliográficas}

Adams, Julia, Clemens Elizabeth, Orloff Ann Shola (2005). Social Theory, Modernity, and the Three Waves of Historical Sociology. En Adams, J.; Clemens, E. y Orloff A. S. (Eds.). Remaking Modernity: Politics, History and Sociology. Duke University Press Books. Estados Unidos.

Ansaldi, Waldo (2017). Arregladitas como para ir de boda. Nuevo ropaje para las viejas derechas. Theomai. No. 35 (primer semestre). Argentina (Pp. 22-51).

Ansaldi, Waldo (1995). Un caso de ficción de organización partidaria o la política sin partidos: Brasil, 1889-1945. Secuencia. Revista de Historia y Ciencias Sociales, Nueva Época. No. 32 (Mayo-Agosto). Instituto Mora. México (Pp. 5794).

Ansaldi, Waldo y Giordano, Verónica (2012). América Latina. La construcción del orden. Ariel. Argentina.

Boschi, Renato y Diniz, Eli (2004). Empresários, interesse e mercado: dilemas do desenvolvimento no Brasil. Editora de la UFMG/Iuperj. Brasil. 
Radiografía de los gabinetes ministeriales en Brasil y Perú (2016-2018). Un análisis comparativo

Bresser Pereira, Luiz Carlos (2006). O novo desenvolvimentismo e a ortodoxia convencional. São Paulo em Perspectiva. Volumen 20, No. 3 (JulioSeptiembre). Brasil (Pp. 5-24).

Bresser Pereira, Luiz Carlos (2012). Brasil, sociedade nacional-dependente. Novos Estudos. No. 93 (Julio). Brasil (Pp. 101-121).

Bresser Pereira, Luiz Carlos (2017). La nueva teoría desarrollista: una síntesis. Economía UNAM. Volumen 40, No. 14 (Enero-Abril). México (Pp. 48-66).

Bresser-Pereira, Luiz Carlos y Diniz, Eli (2009). Empresariado Industrial, Democracia e Poder Político. Novos Estudos. No. 84 (Julio). Brasil (Pp. 83-99).

Canzani, Agustín (2016). En Uruguay hay una nueva derecha con una visión gerencialista de la política. Nueva Sociedad. Edición Digital, Diciembre. Disponible en: http://nuso.org/articulo/agustin-canzani-en-uruguay-hay-unanueva-derecha-con-una-vision-gerencialista-de-la-politica. Consulta 08/4/2019.

Cardoso, Fernando Henrique ([1972] 1993). O Modelo Político Brasileiro e outros ensaios. DIFEL. Brasil.

Castellani, Ana (2008). Ámbitos privilegiados de acumulación. Notas para el análisis del caso argentino (1976-1989). Apuntes de Investigación del CECYP. No.14. Argentina (Pp. 139-157).

Castellani, Ana (2016). La evolución de la élite económica en la Argentina de los años noventa. En Castellani, A. (ed.), Radiografía de la elite económica argentina. UNSAM edita. Argentina.

Castellani, Ana (2018). Lobbies y puertas giratorias Los riesgos de la captura de la decisión pública. Nueva Sociedad. No. 276 (Julio-Agosto). Argentina (Pp. 4861).

Codato, Adriano y Franz, Paulo (2017). Recrutamento ministerial no Brasil: comparando as presidências de FHC e Lula. En E-Legis. Volumen 22, No. 10 (Enero-Abril). Brasil (pp. 44-62).

Codato, Adriano y Franz, Paulo (2018). Technical-ministers and politician-ministers during PSDB and PT presidencies. Brazilian journal of public administration. Volumen 52, No. 5 (Septiembre-Octubre). Brasil (Pp. 776-796).

Codato, Adriano; Cavalieri, Marco; Perissinotto, Renato; Dantas, Eric Gil (2016). Economic mainstream and power: a profile analysis of Central Bank directors during PSDB and PT governments in Brazil. Nova Economía. Volumen 26, No. 3. Brasil (Pp. 687-720).

Codato, Adriano; Massimo, Lucas; Costa, Luiz Domingos (2017). Social positions and political recruitment: A study of Brazilian senators. Tempo Social, revista de sociología da USP. Volumen 29, No. 3 (Diciembre). Brasil (Pp. 111-135).

Cotler, Julio (1995). Political Parties and the Problems of Democratic Consolidation in Peru. En Mainwaring, Scott y Scully, Timothy (Eds.) Building Democratic Institutions: Party Systems in Latin America. Standford University Press. 
Estados Unidos.

Cotler, Julio (1998). La articulación y los mecanismos de representación de las organizaciones empresariales. Documentos de trabajo, No. 97, Serie Sociología y Política, No. 17. IEP. Perú.

Cotler, Julio ([1978] 2005). Clases, Estado y Nación en el Perú. IEP. Perú.

Cotler, Julio y Grompone, Romeo (2000). El fujimorismo: ascenso y caída de un régimen autoritario. IEP. Perú.

Crabtree, Jhon y Durand, Francisco (2017). Perú: elites del poder y captura política. PUC, Universidad del Pacífico, Instituto de Estudios Peruanos (IEP). Perú.

D’Araujo, Maria Celina (2009). A elite dirigente do governo Lula. Centro de Pesquisa e Documentação de História Contemporânea do Brasil da Fundação Getúlio Vargas (CPDOC/FGV). Brasil.

D'Araujo, Maria Celina (2011). PSDB e PT e o Poder Executivo. Desigualdade \&

Diversidade, Revista de Ciências Sociais da PUC-Rio. Dossiê Especial, segundo semestre. Brasil (Pp. 65-100).

D’Araujo, Maria Celina (2014). Elites burocráticas, dirigentes públicos e política no Poder Executivo. En D’Araujo, Maria Celina (Org.), Redemocratização e mudança social no Brasil. Fundação Getúlio Vargas (FGV). Brasil.

Dargent, Eduardo (2014). Technocracy and Democracy in Latin America: The Experts Running Government. Cambridge University Press. Estados Unidos. Degregori, Carlos Ivan (2001). La década de la antipolítica. Auge y huida dede Alberto Fujimori y Vladimiro Montecinos. IEP. Perú.

De Imaz, José Luis ([1962] 1965). Los que mandan. Eudeba. Argentina.

De Riz, Liliana (1986). Política y partidos. Ejercicio de análisis comparado: Argentina, Chile, Brasil y Uruguay. Desarrollo Económico. Volumen XXV, No. 100 (Enero-Marzo). Argentina.

Diniz, Eli (1978). Empresário, estado e capitalismo no Brasil: 1930-1945. Paz e Terra. Brasil.

Diniz, Eli ([1997] 2000). Crise, reforma do Estado e governabilidade: Brasil, 19851995. Fundación Getúlio Vargas. Brasil.

Dogan, Mattei y Pahre, Robert (1993). Las nuevas ciencias sociales. La marginalidad creadora. Grijalbo. México.

Durand, Francisco. (2006). Cuando el poder extractivo captura el Estado. Lobbies, puertas giratorias y paquetazo ambiental en Perú. OFAM. Perú.

Durand, Francisco (2010). Empresarios a la presidencia. Nueva Sociedad. No. 225 (Enero-Febrero). Argentina (Pp. 68-85).

Durand, Francisco (2013). Los Romero: fe, fama y fortuna. El Virrey y DESCO. Perú. Durand, Francisco (2017). Los doce apóstoles de la economía peruana: una mirada social a los grupos de poder limeños y provincianos. Fondo Editorial Pontificia Universidad Católica del Perú. Perú. 
Radiografía de los gabinetes ministeriales en Brasil y Perú (2016-2018). Un análisis comparativo

Flores Andrade, Anselmo (2003). Los empresarios y la transición a la democracia: los casos de México y España. Revista mexicana de sociología. Volumen 65, No. 3 (Julio-Septiembre). México (Pp. 497-522).

Furtado, Celso ([1959] 1962). Formación económica de Brasil. Fondo de Cultura Económica (FCE). México.

Furtado, Celso (1965). Dialéctica del desarrollo. Fondo de Cultura Económica (FCE). México.

Furtado, Celso (1992). Brasil: La construcción interrumpida. Fondo de Cultura Económica (FCE). México.

Furtado, Celso (1998). El capitalismo global. Fondo de Cultura Económica (FCE). México.

Giordano, Verónica (2011) Alegato en favor de una Sociología Histórica Comparada para América Latina. Trabajo y Sociedad. No. 17. Argentina (Pp. 41-48). Disponible en:

Giordano, Verónica (2014) ¿Qué hay de nuevo en las "nuevas derechas”? Nueva Sociedad. No. 254 (Noviembre-Diciembre). Argentina (Pp. 46-56).

González Bozzolasco, Ignacio (2018). El nuevo rostro del partido colorado. Nueva Sociedad. Edición Digital, Abril. Disponible en: http://nuso.org/articulo/elnuevo-rostro-del-poder-colorado. Consulta 08/4/2019.

Mainwaring, Scott ([1995] 1996). Brasil: Partidos Débiles, Democracia Indolente. En Mainwaring, Scott (Ed.). La Construcción de Instituciones Democráticas.

Sistemas de Partidos en América. CIEPLAN. Estados Unidos.

Mainwaring, Scott (2009). Deficiencias estatales, competencia entre partidos y confianza en la representación democrática en la región andina. En Mainwaring, Scott; Ana María Bejarano; Eduardo Pizarro (Eds.). La crisis de la representación democrática en los países andinos. Norma. Argentina.

Malpica Santisteban, Carlos (1964). Los dueños del Perú. Persistiremos. Perú.

Martuscelli, Danilo (2013). Crises políticas e capitalismo neoliberal no Brasil. Tesis

Doctoral. Instituto de Filosofía y Ciencias Humanas, Universidad Estadual de Campinas. Brasil.

Martuscelli, Danilo (2018). Burguesia interna e capitalismo dependente: uma reflexão a partir dos casos argentino e brasileiro. Crítica Marxista. No. 47. Fundação Editora da UNESP. Brasil (Pp. 55-74).

Meléndez, Carlos (2012). Partidos inesperados. La institucionalización del sistema de partidos en un escenario de post colapso partidario. Perú 2001-2011. Friedrich Ebert Stiftung. Perú.

Meneguello, Rachel (2002). El impacto de la democratización del Estado en el desarrollo de los partidos brasileños (1985-1998). En Cavarizzi, Marcelo y Abal Medina, Juan (h) (Comps.). El asedio a la política. Los partidos latinoamericanos en la era neoliberal. Homo Sapiens Ediciones. Argentina. 
Perissinotto, Renato; Dantas, Eric Gil; Codato, Adriano; Filipi, Thais M. (2015). Ministros de Economía, burocracia y política económica en el desarrollismo brasileño (1930-1964). En Anuario Centro de Estudios Económicos de la Empresa y el Desarrollo (CEEED). Volumen 7, No. 7. Facultad de Ciencias Económicas (FCE). Argentina (Pp. 57-102).

Rodríguez, Gina Paola (2014). Álvaro Uribe y Juan Manuel Santos: ¿una misma derecha? Nueva Sociedad. No. 254 (Noviembre-Diciembre). Argentina (pp. 8499).

Rubio, Julia María (2016). El sistema de partidos de Perú (1980-2015). En Friedenberg, Flavia (Ed.) Los sistemas de partidos en América Latina (1978-2015). UNAM-INE. México.

Salas Oroño, Amílcar (2016). Brasil 2016: del presidencialismo de coalición al golpismo. En Prácticas de oficio. No. 17 (Agosto). Argentina (Pp. 30-36).

Singer, André (2009). Raízes sociais e ideológicas do lulismo. Novos Estudos. No. 85 (Noviembre). Brasil (Pp. 83-102).

Singer, André (2012) Os sentidos do lulismo. Reforma gradual e pacto conservador. Companhia das letras. Brasil.

Singer, André (2015). Cutucando onças com varas curtas - O ensaio desenvolvimentista no primeiro mandato de Dilma Rousseff (2011-2014). Novos Estudos. No. 102 (Julio). Brasil (Pp. 43-71).

Soler, Lorena (2018). Elecciones en Paraguay: ¿Game over para la democracia? Panamá. Edición Digital, Abril. Disponible en: http://panamarevista.com/elecciones-en-paraguay-game-over-para-latecnocracia. Consulta 08/4/2019.

Tanaka, Martin (2005). Democracia sin partidos. Perú 2000-2005. IEP. Perú.

Tanaka, Martin (2010). La dinámica "neodualista" de una democracia sin sistema de partidos: la situación de la democracia en el Perú. Revista de Ciencia Política. Volumen 30, No. 1. Perú (Pp. 87-114).

Távara, José Ignacio (2004). Captura del Estado, persistencia de la corrupción y desafíos a la democracia. Seminario Internacional sobre Corrupción y Vicios Públicos. Perú.

Thorp, Rosemary; Zeballos, Graciela (2001). Las políticas económicas del régimen de Fujimori: ¿un retorno al pasado? ECONOMIA Revista del Departamento de Economía Pontificia Universidad Católica del Perú. Vol. 24, No. 47 (junio). Perú (Pp. 9-42).

Tilly, Charles (1991). Grandes estructuras, procesos amplios, comparaciones enormes. Alianza Editorial. España.

Trindade, Hélgio (1986). La construcción del Estado nacional en Argentina y Brasil (1810-1900). Revista Mexicana de Sociología. Volumen XLVIII, No. 1 (eneromarzo). México (Pp. 137-166). 
Radiografía de los gabinetes ministeriales en Brasil y Perú (2016-2018). Un análisis comparativo

Tuesta Soldevilla, Fernando (Ed.) (2016). Perú: elecciones 2016. Un país dividido y un resultado inesperado. PUC. Lima.

Vásquez, Enrique (2000). Estrategias del poder. Grupos económicos en el Perú. Universidad del Pacífico. Perú.

Viguera, Aníbal (1996). Empresarios y acción política en América Latina. Una perspectiva comparada. Nueva Sociedad. No. 143 (Mayo-Junio). Argentina (Pp. 174-189).

Vommaro, Gabriel (Ed.) (2015). Mundo Pro. Anatomía de un partido fabricado para ganar. Planeta. Argentina.

Vommaro, Gabriel (2017). La larga marcha de cambiemos. Siglo XXI editores. Argentina.

Wright Mills, Charles ([1956] 1963). La elite de poder. Fondo de Cultura Económica (FCE). México. 
Inés Nercesian y Roberto Cassaglia

Telos Vol. 21, No. 2 (2019). 372-400

\section{Anexos}

Cuadro 2. Carreras de grado

\begin{tabular}{|lc|c|c|c|c|c|}
\hline & \multicolumn{2}{c}{ Brasil } & \multicolumn{2}{c|}{ Perú } & \multicolumn{2}{c|}{ Total general } \\
\cline { 2 - 7 } $\begin{array}{l}\text { Abogacía y } \\
\text { ciencias }\end{array}$ & & & & & & \\
jurídicas & 30 & $45,5 \%$ & 8 & $17,8 \%$ & 38 & $34,2 \%$ \\
$\begin{array}{l}\text { Economía } \\
\text { Ingeniería }\end{array}$ & 7 & $10,6 \%$ & 15 & $33,3 \%$ & 22 & $19,8 \%$ \\
$\begin{array}{l}\text { Administración } \\
\text { de empresas }\end{array}$ & 7 & $12,1 \%$ & 5 & $11,1 \%$ & 13 & $11,7 \%$ \\
$\begin{array}{l}\text { Sociología y } \\
\text { ciencias }\end{array}$ & & $10,6 \%$ & 2 & $4,4 \%$ & 9 & $8,1 \%$ \\
humanas & 3 & $4,5 \%$ & 6 & $13,3 \%$ & 9 & $8,1 \%$ \\
Otros & 3 & $4,5 \%$ & 2 & $4,4 \%$ & 5 & $4,5 \%$ \\
Medicina & 2 & $3,0 \%$ & 2 & $4,4 \%$ & 4 & $3,6 \%$ \\
$\begin{array}{l}\text { Educación } \\
\text { Psicología }\end{array}$ & 3 & $1,5 \%$ & 2 & $4,4 \%$ & 3 & $2,7 \%$ \\
Defensa y & & $4,5 \%$ & & & 3 & $2,7 \%$ \\
$\begin{array}{l}\text { Seguridad } \\
\text { Arte y cultura }\end{array}$ & & $0,0 \%$ & 2 & $4,4 \%$ & 2 & $1,8 \%$ \\
Comunicación & & $0,0 \%$ & 1 & $2,2 \%$ & 1 & $0,9 \%$ \\
y medios & 1 & $1,5 \%$ & & & & \\
No realizó & 1 & $1,5 \%$ & & & 1 & $0,9 \%$ \\
Total general & 66 & $100 \%$ & 45 & $100 \%$ & 111 & $0,9 \%$ \\
\hline
\end{tabular}

Fuente: elaboración propia en base a datos del Observatorio 


\section{Radiografía de los gabinetes ministeriales en Brasil y Perú (2016-2018). Un análisis comparativo}

Cuadro 7. Ocupación anterior al cargo

\begin{tabular}{|c|c|c|c|c|c|c|}
\hline \multirow[b]{2}{*}{ Procedencia } & \multicolumn{3}{|r|}{ Brasil } & \multicolumn{3}{|r|}{ Perú } \\
\hline & Cantidad & $\%$ & & Cantidad & $\%$ & \\
\hline $\begin{array}{l}\text { 1. Administración } \\
\text { Pública }\end{array}$ & 58 & $87,9 \%$ & $\begin{array}{l}\text { Poder Ejecutivo (31), Legislativo (20), Judicial (1), Entes } \\
\text { autárquicos estatales (6) }\end{array}$ & 26 & $57,8 \%$ & $\begin{array}{c}\text { Poder Ejecutivo (15), Legislativo (6), Entes autárquicos } \\
\text { estatales (5) }\end{array}$ \\
\hline $\begin{array}{l}\text { 2. Sector Privado y } \\
\text { Tercer Sector }\end{array}$ & 8 & $12,1 \%$ & & 19 & $42,2 \%$ & \\
\hline $\begin{array}{l}\text { 2.1. Actividades } \\
\text { Financieras y } \\
\text { energéticas }\end{array}$ & 2 & $3,0 \%$ & Banco Itaú (1), J\&F Holding (1) & 4 & $8,9 \%$ & $\begin{array}{l}\text { Thorne \& Associates/ Bolsa de Valores de Lima (1), } \\
\text { Empresa Hidroeléctrica Pucamarca SAC (1), Macroconsult } \\
\text { (1), Banco Internacional del Perú (Interbank) (1) }\end{array}$ \\
\hline \begin{tabular}{|l|} 
2.2. Actividades \\
Profesionales y \\
consultorías (Jurídicas \\
y de Contabilidad, de \\
Consultoría en Gestión \\
Empresarial y \\
Publicidad e \\
Investigación de \\
Mercados) \\
\end{tabular} & 6 & $9,1 \%$ & $\begin{array}{l}\text { A2B Consultoria Empresarial Ltda. (1), Pereira, Moraes e } \\
\text { Oliveira Sociedade de Advogados (1), Escritório de Advocacia } \\
\text { Sergio Bermudes (1), Torquato Jardim Advogados Associados } \\
\text { (1), Medina Osório Advogados (1), Independiente (1) }\end{array}$ & 5 & $11,1 \%$ & $\begin{array}{l}\text { Promotick y Distana (1), Grupo de Análisis para el } \\
\text { Desarrollo Económico (GRADE) (1), Consultoría política } \\
\text { (2), Escuela Superior de Administración y Negocios } \\
\text { (ESAN) (1). }\end{array}$ \\
\hline \begin{tabular}{|l|} 
2.3. Artes, \\
Entretenimiento y \\
Recreación \\
\end{tabular} & & & & 1 & $2,2 \%$ & Dirección de cine y actuación (1) \\
\hline $\begin{array}{l}\text { 2.4. Enseñanza/ } \\
\text { consultorías no } \\
\text { vinculadas al mundo } \\
\text { empresarial }\end{array}$ & & & & 6 & $13,3 \%$ & $\begin{array}{l}\text { Universidad Cayetano Heredia (1), Investigador y consultor } \\
\text { en termas de seguridad ciudadana y conflictos sociales } \\
\text { (1),Universidad San Martín de Porres (1), Investigador, } \\
\text { miembro consultivo y profesor universitario de diversas } \\
\text { instituciones académicas nacionales e internacionales } \\
\text { (1),Centro Kluge de la Biblioteca del Congreso } \\
\text { estadounidense y en el Instituto de Estudios Políticos de } \\
\text { París (1), Gestor en políticas públicas e institucionales, } \\
\text { programas y proyectos sociales y docente universitario (1) }\end{array}$ \\
\hline \begin{tabular}{|l|} 
2.5. Industria \\
Manufacturera
\end{tabular} & & & & 1 & $2,2 \%$ & Backus y Johnston (1), \\
\hline $\begin{array}{l}\text { 2.6. Información y } \\
\text { Comunicación }\end{array}$ & & & & 1 & $2,2 \%$ & Llorente \& Cuenca (1) \\
\hline $\begin{array}{l}\text { 2.7. Otras Actividades } \\
\text { de Servicios en } \\
\text { organizaciones } \\
\text { empresariales, } \\
\text { profesionales y de } \\
\text { empleadores } \\
\end{array}$ & & & & 1 & $2,2 \%$ & $\begin{array}{l}\text { Sociedad de Comercio Exterior del Perú (COMEXPERÚ) } \\
\text { (1) }\end{array}$ \\
\hline
\end{tabular}

